\title{
The prediction of cement energy demand based on support vector machine
}

\author{
Xin Zhang ${ }^{a,{ }^{*}}$, Shaohong Jing ${ }^{b}$ \\ School of Electrical Engineering University of Jinan, Jinan 250022, China \\ a18254136693@163.com, ${ }^{\mathrm{b}} \mathrm{cse}$ jsh@ujn.edu.cn
}

Keywords:support vector regression, cement energy forecast.

\begin{abstract}
Aiming at the energy waste problems resulting from the contradiction between energy demand and supply in cement production, an energy demand prediction model based on support vector regression is built. And energy consumption of a cement enterprises in Shanxi cement production line, for example, to validate the model simulations show that the model has good predictive results.
\end{abstract}

\section{Introduction}

The cement industry has always been one of high energy-consuming industries,whose energy consumption accounts for about $8 \%$ of national energy consumption, while it accounts for more than $40 \%$ of the total cement production and consumption. Now energy shortages and rising energy prices have been restricting the development of the cement industry, so the cement companies must reduce energy consumption to the minimum range as much as possible, so as to reduce the cost of cement production to make the cement have a price advantage in modern industry, and promote the rapid development of the cement industry. It is imperative to predict the energy consumption of the cement production and then control it according to the results.

This article establishes SVM model on the basis of the daily electric energy consumption in April 2014 in Shanxi Province 1\# cement line, and predicts according to the model results, makes simulation experiments using MATLAB software, and compare the performance with the gray prediction GM $(1,1)$, and make a conclusion that SVM can better predict the energy consumption in cement production.

\section{Analysis of Energy Consumption in cement companies}

At present cement production adopts basically new dry production technology. It has largely replaced the out processes, as wet process or kilns, and achieved certain results in terms of increasing the production of cement companies. But, the higher yield also brings higher energy consumption [1]. Depending on the scale of cement production line, there may be differences in cement technology, but basically there will be crushing and pre-homogenization of raw materials, raw material preparation, coal preparation, clinker, cement grinding, cement packing and delivery and so on. Within a certain range, each step of the cement business in energy consumption can directly reflect the scene of the energy consumption of cement. So forecasting the energy consumption of every cement production line can reflect the energy consumption of cement each process to a certain extent and reflect the energy consumed in cement production. The cement company managers can predict the results which based on cement consumptions to find the cause of abnormal consumption and control it. So they can achieve effective development of cement production.

The energy consumption in the cement production process is a power and coal. The two parts accounts for more than $99 \%$ of the total energy consumption of the entire production. This only predicts the electricity consumption of a single step in the cement production process. Every cement production needs electricity. In the present new dry cement production, the electricity that really used for material for grinding is less than $20 \%$ in the total electricity consumption of the cement production process, while the sum of the key facilities of energy consumption on the total cement production process accounts for more than $80 \%$ of the cement production process. Research on the energy 
consumption of the single cement production process enables managers systematically of cement companies understand utilization of energy, in order to find the cause of wasteful energy usage. The final result is that cement companies can provide the data support in the improving the energy efficiency of cement.

\section{Support Vector Machines Basic Introduction}

Support vector machine (Support Vector Machine, SVM) is a new and efficient machine learning based on the statistics theory. It has many advantages compared to neural networks, for example, strong generalization ability, easy to train, no local minimum value and there is no need to pre-determine the network topology. SVM is a new technology of data mining and is primarily by means of optimization methods. So it can successfully deal with regression (Support vector Machine for regression, SVR) and pattern recognition (support vector classification, SVC), and many other question. Besides, the SVM can be extended to the prediction field. At present, the theory research and applied research related to SVM have received extensive attention domestically and internationally. The prediction uses the one of the SVM (Support Vector Machine for Regression, SVR) [2].

There is a little difference of the basic idea between the SVR and SVM. SVM is becoming to look for an optimal classification aspect, so that there is an error minimum from all training samples to the optimal classification aspect. The idea of realizing SVR is to use a non-linear mapping to map data $x$ into a high-dimensional feature space, and then implement linear regression in the space.

\subsection{The establishment of energy demand prediction model.}

Given training set $\left\{\left(x_{i}, y_{i}\right), i=1,2,3, \ldots, n\right\}$, which $n$ represents the quantity of samples, $x_{i}\left(x_{i} \in R^{d}\right)$ represents the input column vector of $i$ training sample, and $y_{i} \in R$ for the corresponding input values. SVR regression function expressions are as follows:

$$
f(x)=\omega \cdot \varphi(x)+b^{(1)}
$$

In the expression, $\varphi(x)$ represents a nonlinear mapping function, $\omega, b$ are the parameters that need to be identified in the system model [3].

Using the structural risk minimization principle, the parameters that need to beidentified in the last formula is handled as following:

$$
R(\omega)=\sum_{i=1}^{n} C\left(e_{i}\right)+\frac{1}{2}\|\omega\|^{2}
$$

In (2) formula above, $R(\omega)$ is called empirical risk, $\|\omega\|^{2}$ called the confidence risk, $C\left(e_{i}\right)$ called the loss function. According the SVR principle, in order to solve to (2) is equal to optimization of (3):

$$
\begin{aligned}
& \min \frac{1}{2}\|\omega\|^{2}+C \sum_{i=1}^{n}\left(\xi_{i}+\xi_{i}^{*}\right) \\
& \text { s.t. }\left\{\begin{array}{c}
y_{i}-\omega \varphi\left(x_{i}\right)-b \leq \varepsilon+\xi_{i} \\
y_{i}-\omega \varphi\left(x_{i}\right)-b \geq \varepsilon+\xi_{i}^{*} \\
\xi_{i} \geq 0, \xi_{i}^{*} \geq 0, i=1,2,3, \ldots, n
\end{array}\right.
\end{aligned}
$$

In which $\varepsilon$ represents for precision parameter of regression function, $C$ for penalty factor, and $\xi_{i}$, $\xi_{i}^{*}$ the relaxation factor.

$$
\begin{aligned}
& \max _{a_{i}, a_{i}^{*}}\left[-\frac{1}{2} \sum_{i, j=1}^{n}\left(a_{i}-a_{i}^{*}\right)\left(a_{j}-a_{j}^{*}\right) K\left(x_{i}, x_{j}\right)+\sum_{i=1}^{n}\left(a_{i}-a_{i}^{*}\right) y_{i}-\varepsilon \sum_{i=1}^{n}\left(a_{i}+a_{i}^{*}\right)\right] \\
& \text { s.t. }\left\{\begin{array}{l}
\sum_{i=1}^{n}\left(a_{i}-a_{i}^{*}\right)=0 \\
a_{i}, a_{i}^{*} \in[0, C]
\end{array}\right.
\end{aligned}
$$


In the above formula, $K\left(x_{i}, x_{j}\right)$ is the core of function. According to the Mercer condition, kernel is function of models choose a Gaussian radial basis kernel functions.

The kernel function is put into the formula, then:

$$
f(x)=\sum_{i=1}^{n}\left(a_{i}-a_{i}^{*}\right) \exp \left(\frac{-\left\|x_{i}-x_{j}\right\|^{2}}{\sigma^{2}}\right)+b
$$

Calculating regression function $f(x)$ comes actually down to the calculation $a_{i}$ and $a_{i}^{*}$. The optimal solution of $a_{i}$ and $a_{i}^{*}$ can be determined by minimizing the conditions.

\subsection{Steps of Concreting Cement Energy Prediction Model Based on Support Vector Machine.}

Construction input and output data.Use of SVM models need to select the training samples and test samples. And sample data comes from the database of cement production, and the database stores the electricity that consumed in the cement production process. Selecting the original data from the database, including the energy consumption data in a different time (days, months, and years) can be used for predicting energy consumption of day, month and year. Then build SVR input and output according to these data.

Data preprocess. The raw data of cement field has a big difference about the value. It may be a big change if directly operated. This will reduce the accuracy of the prediction.So the raw data of database need to be preprocessed. It means data normalization preprocessing using (6).

$$
x=\frac{x_{i}-x_{\min }}{x_{\max }-x_{\min }}(6)
$$

Selection SVR model parameters and performance evaluation of models.SVR prediction model need to determine several parameters before the establishment, namely a Gaussian kernel function parameters $\sigma$ and two free parameters $C$ and $\varepsilon$. Now there is no detailed description of how to select these three parameters in the reference. But they all make the final prediction result deviation as model performance evaluation standard. Select the appropriate parameter values from experience, make repeated cross-validation and then finalize $C=4, \sigma^{2}=0.35$, so $\varepsilon=0.01$. Meanwhile, in order to verify the effectiveness of cement prediction model, daily electric energy consumption of a cement mill line of a cement factory in Shanxi Province in April 2014 is predicted according to the SVR prediction model established.

Getting the Final SVR Model and Predict Energy Consumption of Cement Companies.After repeated experience, we obtained $f(x)$. When determine the energy consumption of cement scene in different days, months, or years, using cement energy consumption prediction model, you can obtain the corresponding cement energy consumption prediction (day, month, and year).

\section{Simulation Experiment}

Select a cement mill line from the database of a Shanxi cement factory, and make the power consumption data within 30 days in April 2014 as the simulation data. Meanwhile, combined with the MATLAB software, we can realize cement energy prediction based on SVM. The cement energy data are as follows:

The above data, a total of 30 data points, can be composed of 28 samples pairs of input and output. The input sample set is as follows:

$\{x(2014.4 .1), x(2014.4 .2), x(2014.4 .3)\},\{x(2014.4 .2), x(2014.4 .3), x(2014.4 .4)\},\{x(2014.4 .3), x(2014.4 .4)$,

$x(2014.4 .5)\},\{x(2014.4 .4), x(2014.4 .5), x(2014.4 .6)\}, \ldots \ldots . . .,\{x(2014.4 .28), x(2014.4 .29), x(2014.4 .30)\}$

The output sample set is as follows:

$\{x(2014.4 .4), x(2014.4 .5), x(2014.4 .6), x(2014.4 .7), x(2014.4 .8), \ldots ., x(2014.4 .30)\}$.

In order to forecast cement mill energy with SVM, we select $25 \%$ data sample set. That is, 8 samples set are test sample set, and 20 samples sets are the training sets. 
Table 1 Electricity Consumption in Shanxi 1 \# cement mill line from 2014.4

\begin{tabular}{c|c|c|c}
\hline Date & $\begin{array}{c}\text { Electricity Consumption (ten } \\
\text { thousand KWH) }\end{array}$ & Date & $\begin{array}{c}\text { Electricity Consumption (ten } \\
\text { thousand KWH) }\end{array}$ \\
\hline 2014.4 .1 & 19.1 & 2014.4 .16 & 19.4 \\
\hline 2014.4 .2 & 23.5 & 2014.4 .17 & 22.57 \\
\hline 2014.4 .3 & 34.8 & 2014.4 .18 & 26.42 \\
\hline 2014.4 .4 & 26.34 & 2014.4 .19 & 38.25 \\
\hline 2014.4 .5 & 28.71 & 2014.4 .20 & 46.01 \\
\hline 2014.4 .6 & 46.8 & 2014.4 .21 & 50.32 \\
\hline 2014.4 .7 & 59.65 & 2014.4 .22 & 52.6 \\
\hline 2014.4 .8 & 48.68 & 2014.4 .23 & 48.1 \\
\hline 2014.4 .9 & 46.59 & 2014.4 .24 & 46.76 \\
\hline 2014.4 .10 & 44.52 & 2014.4 .25 & 42.87 \\
\hline 2014.4 .11 & 42.67 & 2014.4 .26 & 44.34 \\
\hline 2014.4 .12 & 34.54 & 2014.4 .27 & 45.3 \\
\hline 2014.4 .13 & 28.45 & 2014.4 .28 & 46.15 \\
\hline 2014.4 .14 & 38.03 & 2014.4 .29 & 42.37 \\
\hline 2014.4 .15 & 24.38 & 2014.4 .30 & 46.61 \\
\hline
\end{tabular}

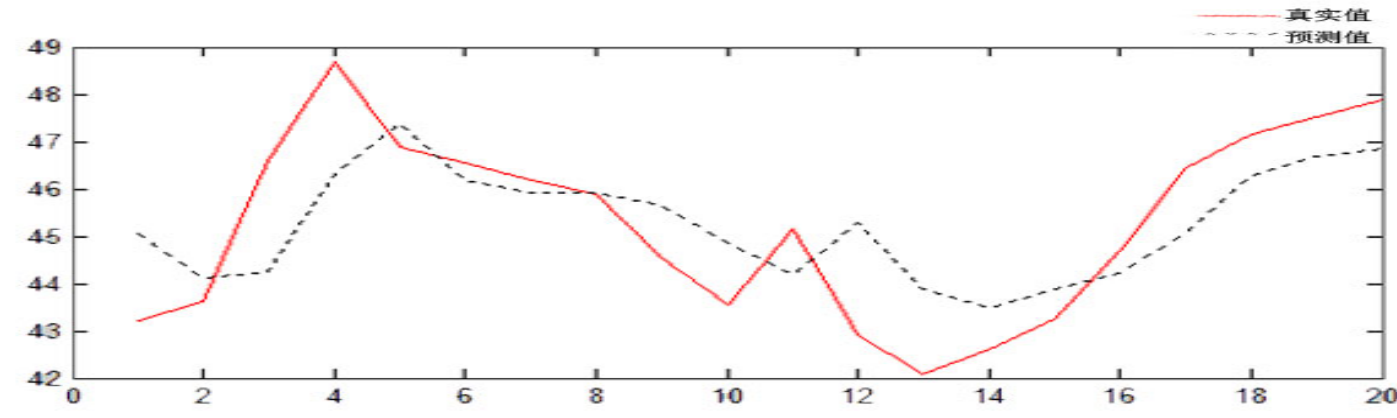

Fig1 SVR training sample set results

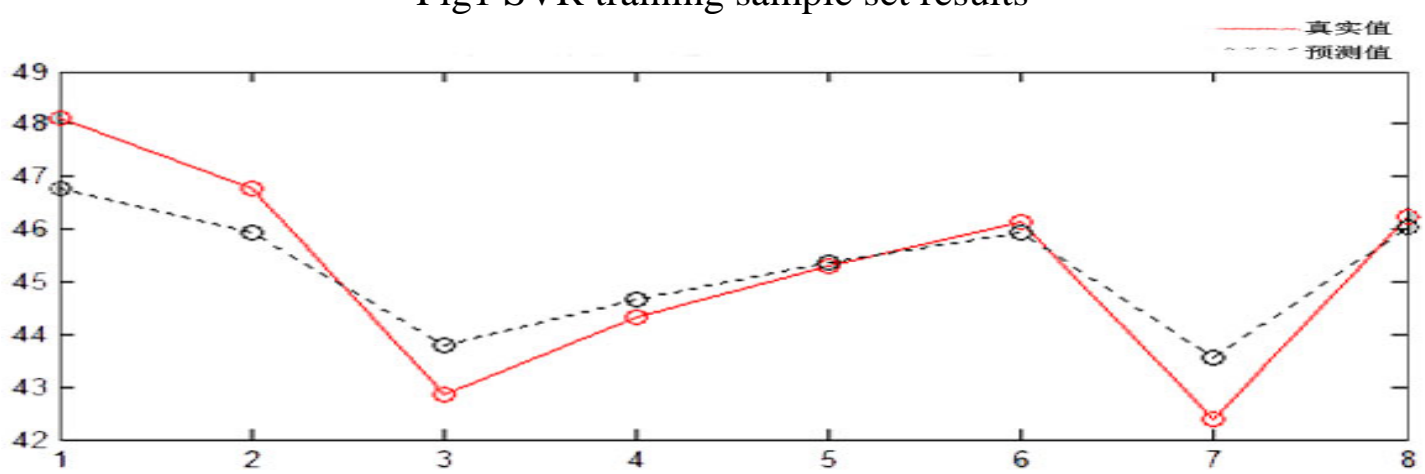

Fig 2 SVR prediction sample set results

It's cement energy prediction resultwhich is shown in Table 2 below:

Table 2 Cement energy prediction result

\begin{tabular}{c|c|c|c|c}
\hline Date & actual value & Predictive value & Relative error & Absolute error \\
\hline 2014.4 .23 & 48.1 & 46.78659 & -1.31341 & $2.7 \%$ \\
\hline 2014.4 .24 & 46.7 & 45.92665 & -0.77335 & $1.65 \%$ \\
\hline 2014.4 .25 & 42.87 & 43.80643 & 0.93643 & $2.2 \%$ \\
\hline 2014.4 .26 & 44.34 & 44.65882 & 0.31882 & $0.72 \%$ \\
\hline 2014.4 .27 & 45.3 & 45.38082 & 0.08082 & $0.17 \%$ \\
\hline 2014.4 .28 & 46.15 & 45.92405 & -0.22595 & $0.48 \%$ \\
\hline 2014.4 .29 & 42.37 & 43.56337 & 1.19337 & $2.8 \%$ \\
\hline 2014.4 .30 & 46.25 & 46.05074 & -0.19926 & $0.43 \%$ \\
\hline
\end{tabular}


From the prediction results above, the errors that forecasting cement energy with SVM makes are within the allowable range. However, the absolute error of training sample is more 0.5078 than the prediction sample. It is only 1.292 and within the range of allowable error.

\subsection{Performance Compare.}

In order to verify the effectiveness and feasibility of SVM forecasting the cement energy, we choose the gray GM $(1,1)$ prediction method that has the greater advantage of small samples and then compare the prediction results [4]. Figure 3 is a gray GM $(1,1)$ prediction result:

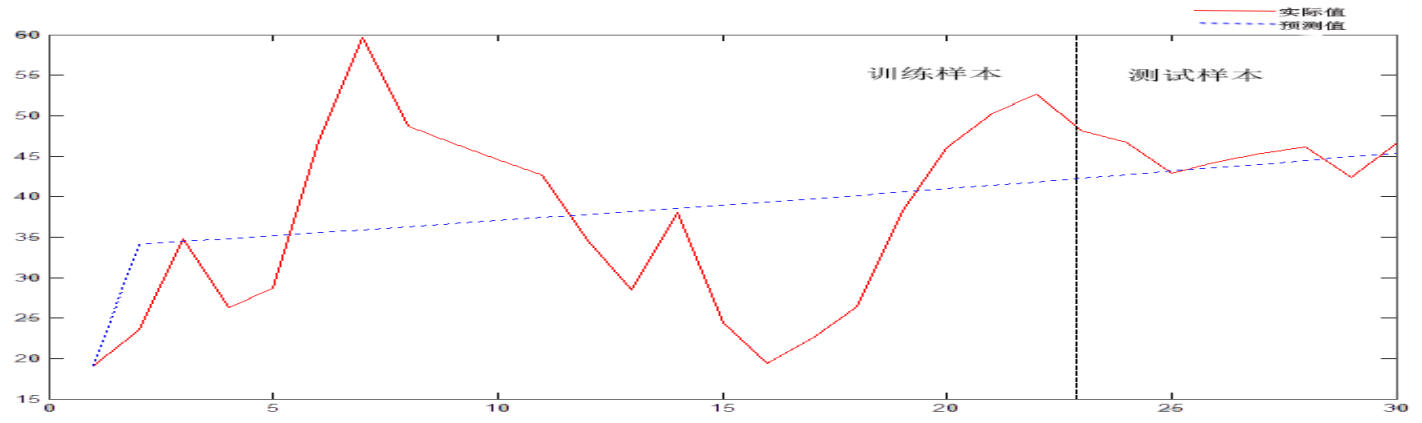

Fig 3 GM $(1,1)$ Prediction Result

The results that cement energy data get with GM $(1,1)$ prediction method is shown in Table 3:

Table3 GM $(1,1)$ predictionresults

\begin{tabular}{c|c|c|c|c}
\hline Date & Actual value & Predictive value & Relative error & Absolute error \\
\hline 2014.4 .23 & 48.1 & 42.2583 & -5.8417 & $12.17 \%$ \\
\hline 2014.4 .24 & 46.7 & 42.6902 & -4.0098 & $8.65 \%$ \\
\hline 2014.4 .25 & 42.87 & 43.1266 & 0.2566 & $0.61 \%$ \\
\hline 2014.4 .26 & 44.34 & 43.5674 & 0.7726 & $1.74 \%$ \\
\hline 2014.4 .27 & 45.3 & 44.0127 & -1.2873 & $2.84 \%$ \\
\hline 2014.4 .28 & 46.15 & 44.4626 & -0.22595 & $0.48 \%$ \\
\hline 2014.4 .29 & 42.37 & 44.9170 & 1.19337 & $2.8 \%$ \\
\hline 2014.4 .30 & 46.25 & 45.3761 & -0.8739 & $1.89 \%$ \\
\hline
\end{tabular}

You can learn from the comparison of the data in Table 2 and Table 3 that, prediction accuracy of support vector machine SVR is better than GM $(1,1)$ prediction accuracy. It is proved that for the cement energy consumption prediction, SVM has more advantages.

\section{Summary}

This article is mainly aimed at energy consumption in the cement production, we build the cement energy demand prediction model. And based on the historical data of cement energy consumption, we make the simulation experiment for the daily electric energy consumption of $1 \#$ cement mill line in a Shanxi Province in April 2014.The results proved that:

SVR cement energy demand prediction model this article used has the good usability. The model overcomes the shortage of traditional prediction method. It has better prediction ability than the gray prediction method, and the prediction accuracy is higher. It is a good reference to reasonably arrange for energy consumption in the cement production process.

Thus, in solving cement energy demand prediction, SVR model has many advantages and is very suitable for the research for the energy consumption prediction in cement production process.

\section{Acknowledgements}

This work was financially supported byMajor projectsofShandong Province independent innovation achievements(2014CGZH0601) and China-EU SMEs Cooperation Fund for energy conservation research project (SQ2013ZOC600003). 


\section{References}

[1]Ceng Xuemin. Cement industry energy consumption status and energy saving potential [J]. China cement, 2006, (3): DOI:10.3969/j.issn.1671-8321.2006.03.006. 16-21.

[2] Yang Wenren. Research and implementation of energy management system based on energy consumption forecasting model [J]. South China University of Technology, 2013

[3]Sun Han, Yang Purong, Cheng Jinhua. Energy demand forecasting model based on Matlab support vector regression model [J]. system engineering theory and practice, 2011, 31 (10): 2001-2007.

[4] Zhou Ruiping. GM $(1,1)$ model of grey prediction method to predict urban population [J]. Journal of Inner Mongolia Normal University: Natural Science Edition, 2005, 34 (1): 81-83. 